\title{
Comunicación de Organizaciones No Lucrativas de la Salud en España: el estado de la cuestión
}

\author{
Hernán Alfredo Díaz \\ Fundación de Educación para la Salud \\ Universidad Complutense de Madrid \\ (Madrid, España)
}

\begin{tabular}{c}
\hline Palabra clave \\
\hline comunicación \\
rganizaciones no lucrativas \\
salud \\
estrategias \\
promoción de la salud \\
participación
\end{tabular}

\begin{abstract}
Resumen
Las Organizaciones No Lucrativas (ONL) han adquirido en la últimas décadas un gran protagonismo en el ámbito de la salud, al punto de que en España hay actualmente más de 6000 organizaciones activas en el campo sociosanitario. Este trabajo analiza el "estado del arte" sobre la investigación de la comunicación de las ONL del ámbito de la salud en España, focalizándose en las organizaciones no sanitarias, a partir de una revisión sistemática de los estudios publicados. La revisión se realizó entre marzo y junio de 2013 y señala que, en términos globales, la investigación en comunicación y salud ha estado focalizada en las relaciones profesionales-pacientes y la información de salud. En España, los estudios sobre comunicación de organizaciones del ámbito de la salud se han centrado tradicionalmente en el objeto de estudio "organizaciones sanitarias", como hospitales y centros de salud, dejando un gran vacío de conocimiento sobre el accionar de las ONL que actúan en el campo sociosanitario. Las principales aportaciones en este campo provienen de estudios genéricos que no refieren al ámbito específico de la salud o que, siendo genéricos, también analizan algunos aspectos puntuales de la comunicación de las ONL de salud. En conclusión, esta revisión revela la necesidad de desarrollar una línea de investigación que estudie específicamente la comunicación de ONL del ámbito de la salud, para avanzar en la gestión profesional desde nuevas perspectivas conceptuales.
\end{abstract}

Hernán Alfredo Díaz forma parte del equipo editorial de Revista de Comunicación y Salud y no ha participado en el proceso de edición de este artículo.

Autor para correspondencia: Hernán A. Díaz. Fundación de Educación para la Salud (FUNDADEPS). Hospital Clínico San Carlos de Madrid, Servicio de Medicina Preventiva, $3^{a}$ Planta Norte. Calle Martín Lagos s/n, 28040, Madrid (España). Correo electrónico: comunicacion@fundadeps.org. Teléfono: (34) 913303422 / 3705 


\section{Non-profit Health Organization's Communications in Spain: state of affair}

\begin{tabular}{c}
\hline Keywords \\
\hline communication \\
non-profit organizations \\
health \\
strategies \\
health promotion \\
participation
\end{tabular}

\section{Cómo citar el artículo}

Díaz Hernán, A. (2014). Comunicación de Organizaciones No Lucrativas de la Salud en España: el estado de la cuestión. Revista de Comunicación y Salud, Vol. 4, pp. 35-48. DOI: http://doi.org/10.35669/revistadecomunicacionysalud.2014.4.35-48 


\section{Introducción}

En la segunda mitad del siglo XX, a partir de la evolución del concepto de salud desde una concepción limitada a un fenómeno físico de "ausencia de enfermedad" a un enfoque bio-psico-social que la definió como "el estado de completo bienestar físico, psíquico y social", se produjo una profunda transformación en las políticas de salud (OMS, 2006). Una de las principales consecuencias de esa evolución conceptual fue que la salud dejó de ser un tema de competencia prácticamente exclusiva del sistema sanitario, al tiempo que se desarrollaban nuevas estrategias de promoción de la salud y educación para la salud que empezaron a poner el énfasis en el control de los determinantes sociales de la salud, especialmente en lo referido a los entornos físicos y sociales en los que viven las personas y a los hábitos estilos de vida que adoptan (Lalonde, 1974; OMS, 1986).

En ese contexto, la sociedad civil organizada comenzó a adquirir un mayor protagonismo en la salud pública a través de asociaciones profesionales y de pacientes, fundaciones, sociedades científicas, redes y plataformas. El proceso de crecimiento y consolidación de estas organizaciones estuvo basado en su capacidad para convertirse en representantes de los intereses de los profesionales, los pacientes y las familias, así como en una incansable tarea que facilitó el acceso de toda la sociedad a más y mejores recursos para la atención, la formación y la educación de las personas. La emergencia de estos nuevos actores productores de salud ha sido objeto de lecturas diversas: para algunos, se trata de procesos de "construcción de alianzas estratégicas" que ampliaron las posibilidades de participación de la sociedad civil (OMS, 2001), y para otros es la respuesta social a las políticas de corte neoliberal que propiciaban el paso del Estado a un segundo plano (Missoni, 2012).

En España, la participación de las Organizaciones No Lucrativas (ONL) del ámbito de la salud ha crecido de forma significativa en las últimas décadas. Según consta en los registros del Ministerio del Interior (2012), en el año 1989 había en España 149 asociaciones que tenían como actividad principal el abordaje de "deficiencias y enfermedades", cifra que creció hasta las 869 en el año 2011; de la misma forma, las organizaciones dedicadas a la "acción sanitaria, educativa y social" pasaron en ese período de 283 a 5328. En el caso de las federaciones, en ese período de tiempo pasaron de 14 a 77 en la primera categoría, y de 10 a 162 en la segunda. Para tener una dimensión aproximada de la importancia social de estas organizaciones, vale decir que en España actualmente hay alrededor de 29.000 organizaciones no lucrativas y el ámbito de la salud es uno de los más importantes: el $22,1 \%$ de las ONL activas en España en el año 2010 tenían como campo de actuación principal el socio-sanitario, solo por detrás de la acción social $(38,6 \%)$ y la integración e inserción $(23,2 \%)$ (Fundación Luis Vives, 2012).

La comunicación adquiere para estas organizaciones un carácter estratégico porque se relaciona, entre otras cuestiones, con su capacidad para posicionar determinada temática en la agenda pública; dar visibilidad a sus acciones; construir su imagen pública; posicionarse como referente e interlocutor ante los otros actores sociales; construir redes con otras organizaciones; y dar cuenta a la sociedad de forma 
transparente de sus actuaciones (Vernis, 1998; Canel y Echart, 2005; Herranz de las Casas, 2007; Balas, 2012; Fundación Luis Vives; 2012).

El objetivo de este trabajo es analizar el "estado del arte" sobre la investigación de la comunicación de las ONL del ámbito de la salud en España a partir de una revisión de los estudios publicados hasta el momento, para identificar con mayor precisión los alcances y limitaciones de esos antecedentes.

\section{Definiciones en torno a las Organizaciones No Lucrativas del ámbito de la Salud}

Antes de adentrarnos en el análisis del estado de arte sobre la comunicación de ONL del ámbito de la salud, es importante establecer con claridad a qué tipo de organizaciones nos estamos refiriendo.

Según el enfoque teórico elegido, estas organizaciones suelen recibir diferentes denominaciones: "organizaciones de la sociedad civil", "organizaciones no gubernamentales", "organizaciones no lucrativas" y "Tercer Sector "son algunas de las más comunes. A los fines de este trabajo, consideramos que la denominación más apropiada es la de "Organizaciones No Lucrativas" (ONL), siguiendo la definición aportada por Vernis y otros (1998): "una entidad constituida para prestar un servicio que mejore o mantenga la calidad de vida de la sociedad; formada por un grupo de personas que aporta su trabajo voluntario; no dedicada al lucro personal de ninguno de sus miembros/socios/fundadores; y que no tiene carácter gubernamental" $(1998,32)$. Esta definición es equiparable a la de "Tercer Sector de Acción Social" (TSAS) incluida en el Anuario de la Fundación Luis Vives (2012; 13).

Salamón (2002) plantea cinco rasgos que definen a las ONL:

- Están organizadas formalmente, con existencia jurídica y una estructura institucional;

- Son privadas, en el sentido de que estas entidades tienen una existencia institucionalmente separada del sector público;

- No tienen ánimo de lucro, lo que se manifiesta en el hecho de que no reparten beneficios para sus gestores o asociados y que los recursos con los que cuentan deben destinarse íntegramente a los fines definidos en los estatutos de la organización;

- Son autónomas, porque controlan de forma independiente sus propias actividades a través de los órganos de gobierno de los que están dotadas;

- La participación es voluntaria, tanto porque los miembros que en ella participan no están obligados a hacerlo por el tipo de actividad que realizan como por el hecho de que muchos de sus miembros participan por su interés en colaborar de forma altruista para el cumplimiento de los fines sociales de la organización. 
Es importante señalar que se trata de un colectivo muy diverso en cuanto a fines, alcances y objetivos, en el que se incluyen organizaciones tanto de primer nivel (asociaciones de profesionales y pacientes, fundaciones, sociedades científicas) como de segundo nivel (plataformas, coordinadoras, redes y federaciones). Se trata de organizaciones que tienen como campo de actuación el sociosanitario, trascendiendo los límites de lo sanitario desde perspectivas que ponen el énfasis en la promoción del bienestar y la calidad de vida de las personas.

\section{Metodología}

Esta investigación esta basada en una revisión profunda y sistemática de la bibliografía relacionada con la comunicación de ONL del ámbito de la salud. El proceso de documentación se realizó entre marzo y junio de 2013, utilizando inicialmente el motor de búsqueda Summon, integrado en la Biblioteca de la Universidad Complutense (http://biblioteca.ucm.es).

La búsqueda inicial se hizo con las siguientes palabras clave (en español e inglés): comunicación + salud (health communication); comunicación + organizaciones + salud (communication + organizations + health); comunicación + organizaciones no lucrativas + salud (communication + non-profit organizations + health).

Posteriormente, se seleccionaron los resultados considerados relevantes para la investigación con los siguientes criterios: en primer lugar, que en el título o la descripción del documento hallado incluyera todas las palabras clave; si no se cumplía el criterio anterior, que el título incluyera alguna de las palabras clave y el resumen o sinopsis sugiriera que su contenido era relevante para la investigación.

En una segunda fase, se realizó una búsqueda específica en las revistas científicas específicas del campo de la comunicación y la salud: Health Communication; Journal of Health Communication; Revista de Comunicación y Salud; y Revista Española de Comunicación Sanitaria. La búsqueda estuvo dirigida a artículos que refirieran a la gestión de la comunicación de organizaciones de salud, incluyendo tanto las organizaciones sanitarias (hospitales o centros de salud) como las ONL sociosanitarias estudiadas en este trabajo. Se prestó especial atención a los artículos de revisión publicados en las dos revistas anglosajonas, que sistematizaban las tipologías, temáticas y enfoques de los artículos publicados en las últimas dos décadas.

\section{El estado de la cuestión}

El cruce de la comunicación y la salud se constituyó como campo de estudio en las últimas décadas del siglo XX. En los países anglosajones, que han sido los impulsores a nivel académico de este campo de estudio, las investigaciones se desarrollaron en torno al concepto de health communication. En cambio, en el ámbito iberoamericano se carece todavía de un concepto único y se utilizan diferentes expresiones, como "comunicación y salud" (Cuesta y otros, 2008; Terrón, 2010; Petracci y Waisbord, 2011), "comunicación para la salud (Coe, 1998; Díaz, 2011) o "comunicación en salud" (Muñoz-Cruzado y Barba, 2010; Obregón, 2010). De todos ellos, el de "comunicación 
y salud" parece ser el que se ha ido imponiendo en el ámbito académico, como punto de cruce entre esos dos ámbitos de investigación.

Ugarte (2008) y Terrón (2010) han señalado que la investigación en comunicación y salud tiene una sólida raíz anglosajona y un desarrollo más reciente y todavía embrionario en el ámbito iberoamericano. Así lo demuestran el hecho de que las dos principales publicaciones de este campo de estudio, Health Communication y Journal of Health Communication, se publican en lengua inglesa. Más recientemente han nacido dos publicaciones en español, Revista Española de Comunicación en Salud (en español) y Revista de Comunicación y Salud (que también incluye artículos en portugués).

Como han señalado varios autores, los primeros planteamientos en comunicación y salud se realizaron desde una perspectiva instrumental de la comunicación, que la concebía como un proceso de transmisión unidireccional de información y conocimientos con fines educativos y persuasivos, desde las fuentes de saber a los individuos y la sociedad en general para que estos adoptaran determinadas actitudes y conductas (Coe, 1998; Hill, 2008; Díaz, 2011; Petracci y Waisbord, 2011).

Planteamientos posteriores han ampliado esa concepción de la comunicación en un sentido estratégico y relacional, en procesos de interacción y construcción social de sentido basados en el reconocimiento de los diferentes actores implicados en los procesos de salud y sus particularidades sociales y culturales (OPS, 2001; Instituto Nacional del Cáncer, 2004; Cuesta y otros, 2008; Díaz y Uranga, 2011; Díaz, 2011).

Nos encontramos, por tanto, ante un campo de enorme complejidad, construido desde la interdisciplinariedad y la transdisciplinariedad (Kreps y Maibach, 2008; Obregón, 2010; Terrón, 2010; Medina y González, 2011), en el que podemos identificar diversas líneas de investigación:

- Las relaciones entre profesionales sanitarios y los pacientes y sus familias.

- La información en salud, que incluye tanto el periodismo especializado en salud como la divulgación de la ciencia.

- La publicidad y el marketing de la salud.

- La comunicación en clave comunitaria, en la que se incluyen las campañas y programas de prevención de la enfermedad, promoción de la salud y educación para la salud.

- La comunicación de riesgos sanitarios, incluida la gestión de crisis.

- La e-salud, devenida del desarrollo e implantación de las TIC en el ámbito de la salud. 
- La comunicación de las organizaciones de salud, tanto de las organizaciones sanitarias (hospitales, centros de salud) como de otras organizaciones que se desempeñan en este campo (asociaciones, fundaciones, federaciones).

Esta revisión está centrada en esta última rama de estudio dentro del campo de la comunicación y la salud, poniendo el foco sobre las organizaciones no sanitarias. Inicialmente haremos referencia a los antecedentes desde una visión genérica de la investigación en comunicación y salud, para posteriormente centrarnos en los estudios específicos sobre comunicación de ONL del ámbito de la salud.

\subsection{Las relaciones profesionales-pacientes y la información de salud, principales focos de la investigación en comunicación y salud}

La búsqueda realizada permite afirmar que es escasa la literatura científica sobre comunicación y salud publicada por fuera de las revistas especializadas en ese campo, en línea con las conclusiones a las que arribaron Beck y otros (2004) en un estudio que analizó 18 publicaciones científicas dedicadas a la comunicación (entre ellas Health Communication y Journal of Health Communication) y una de investigación cualitativa en salud (Qualitative Health Research). Ese estudio mostró que el $75 \%$ de los artículos publicados en el período de estudio (1989-2000) se concentraban en las dos revistas especializadas y en la de investigación cualitativa en salud y que solo el $4 \%$ ciento de los artículos de las revistas de comunicación generalistas estaban dedicados a cuestiones de salud.

El estudio liderado por Beck permitió identificar las áreas o temas de comunicación y salud más publicados en esas revistas científicas: la información en salud (13\%), las campañas $(12 \%)$, la relación entre profesionales sanitarios y pacientes $(11 \%)$; el apoyo social (8\%) y los aspectos tecnológicos (2\%). De forma agregada, las categorías relacionadas con información en salud, campañas y medios de comunicación representaban el 35\% de los artículos, y las referidas a las relaciones entre profesionales sanitarios y pacientes el $26 \%$, lo que muestra el peso de ambas líneas de investigación.

Evans (2009) realizó entre 2004 y 2009 un seguimiento de la bibliografía sobre comunicación y salud, a partir de una monitorización de más de un centenar de publicaciones científicas de comunicación, información científica, salud pública, medicina y psicología. Esta monitorización, que se publicó regularmente en Health Communication, clasificó las nuevas publicaciones sobre comunicación y salud en lengua inglesa en seis categorías: campañas de promoción de la salud; teorías e investigación en comunicación y salud; comunicación y salud en los medios; comunicación médico-paciente, comunicación en contextos médicos y comunicación interpersonal; información en salud e informática; y comunicación de riesgos. La propia categorización y el volumen de artículos que aparecen en cada una de las categorías en los sucesivos artículos señalan dos prioridades en la investigación en este campo: el ámbito sanitario, tanto en lo referido a las relaciones entre médicos y pacientes como a la comunicación de las organizaciones de asistencia sanitaria, obviando la comunicación de organizaciones no lucrativas tal y como las hemos definido en este trabajo; y la comunicación de la salud en los medios, con un claro énfasis informativo. 
Un estudio de Freimuth y otros (2006) sobre los artículos publicados por Journal of Helth Communication en sus primeros diez años de vida indica que el $60 \%$ de ellos tenía algún tipo de relación con los medios de comunicación (información de salud, campañas en medios masivos, publicidad, eduentretenimiento), y un $23 \%$ abordaba aspectos relacionados con la relación entre profesionales sanitarios y pacientes; no se aportaba información alguna sobre el $17 \%$ restante.

En un análisis de los artículos publicados en los primeros 75 números de Health Communication, Thompson (2006) señaló que el 20,7\% referían a la comunicación entre profesionales sanitarios y pacientes; el $13,4 \%$ a campañas; el 11,8\% a comunicación de riesgos; el 8,4\% a envejecimiento; el $7 \%$ al uso del lenguaje y el 5,9\% a la información de salud en los medios.

Similares resultados mostró un estudio de Kim y otros (2010) sobre los 642 artículos publicados por Health Communication en sus primeros 22 años de vida. A través de un análisis cualitativo, señaló que el foco de esos estudios está puesto de forma prioritaria en el estudio de las audiencias (41,4\%), la interacción entre profesionales y pacientes $(15 \%)$, los mensajes $(13,1 \%)$ y los emisores $(6,2 \%)$. En esta última categoría podrían estar incluidas las organizaciones del ámbito de la salud, pero como no se dan mayores detalles y a la luz de los estudios previos es posible concluir que hace referencia a organizaciones sanitarias.

Recientemente, una revisión realizada por Nazione y otros (2013) sobre los artículos originales publicados en el período 2000-2009 por las revistas Health Communication y Journal of Health Communication coincide con los estudios previos en que los principales temas de investigación fueron el rol de los medios de comunicación $(22,9 \%)$ y las relaciones entre profesionales sanitarios y pacientes $(13,8 \%)$, pero señala a la vez que el estudio del uso de Internet y las nuevas tecnologías de la comunicación es ya el tercer tema de investigación (13,1\%). De forma agregada, se constata que la difusión de información sobre temas de salud $(45,9 \%)$ y los temas relacionados con los trabajadores sanitarios $(26,9 \%)$, entre ellos la relación con los pacientes, representan los campos de investigación predominantes.

El análisis de publicaciones sobre investigaciones realizadas en España refuerzan la idea de que los estudios sobre comunicación de organizaciones del ámbito de la salud se han centrado tradicionalmente en el objeto de estudio "organizaciones sanitarias", como hospitales y centros de salud, dejando un gran vacío de conocimiento sobre el accionar de las ONL que desempeñan en este campo. Algunas investigaciones se han centrado en la comunicación de los hospitales, desde conceptos diversos como los de marketing (Errasti, 2000), estrategia (Medina, 20121), responsabilidad social corporativa (Medina, 20122) y gestión del conocimiento (Costa, 2011). Investigadores de la Escuela Andaluza de Salud Pública liderados por March han estudiado la comunicación interna de los hospitales (March y otros, 2000). Estudios similares se han realizado respecto de la comunicación interna de centros de atención primaria (Ballvé y otros, 2008; March y otros, 2011; Bustamente, 2013), incorporando elementos que refieren tanto a los procesos y canales de comunicación como a la motivación y satisfacción de los empleados. Saló y Cleriés (2007) abordaron la auditoría de 
comunicación como una función directiva para la gestión del cambio y la adaptación a los entornos interno y externo.

\subsection{Un punto de partida: la investigación sobre comunicación de organizaciones no lucrativas}

Un análisis detallado de la literatura científica disponible nos indica la práctica ausencia de investigaciones previas que aborden de forma específica y en profundidad el objeto de estudio de este trabajo: la comunicación de organizaciones no lucrativas del ámbito de la salud. Las principales aportaciones provienen de estudios genéricos que no refieren al ámbito específico de la salud, como la investigación realizada por Montserrat Balas (2012) sobre la gestión de la comunicación en el Tercer Sector, o que siendo genéricos también analizan algunos aspectos específicos de la comunicación de las ONL de salud, como el Anuario del Tercer Sector de Acción Social de la Fundación Luis Vives (2012).

Aunque la investigación de Balas también incluye a organizaciones de la economía social y ofrece unos resultados generales en los que no se desagregan los correspondientes a las organizaciones del ámbito de la salud, aporta datos novedosos sobre la gestión de la comunicación de las ONL. Afirma que la comunicación es un pilar estratégico de estas organizaciones, que tiene objetivos tan diversos como aumentar el conocimiento; construir una imagen pública alineada con su identidad; y mejorar la notoriedad y el posicionamiento del sector, con el fin último de aumentar el reconocimiento por parte de los distintos grupos de interés (opinión pública, administraciones, empresas, medios de comunicación). Su investigación señala que los directivos de las ONL coinciden con esta visión estratégica, aunque eso luego no se traduce en la asignación de los recursos necesarios: el $52 \%$ de las ONL dedican a la gestión de la comunicación menos del $2 \%$ del presupuesto global y el $7 \%$ no le dedica financiación alguna; el $57 \%$ de las organizaciones tiene un responsable de comunicación, pero solo en una cuarta parte de los casos tiene dedicación a tiempo completo, y en muchos casos compatibiliza estas actividades con responsabilidades en otras áreas de gestión. Balas también señala el desacuerdo en las percepciones de los gestores de las organizaciones y sus dircoms: los primeros dicen considerarla una herramienta estratégica, pero los dircoms se quejan de que no se le otorga suficiente importancia.

El mismo estudio indica que las ONL atribuyen máxima importancia a obtener notoriedad y construir imagen de marca (43\%) y mejorar la reputación de la entidad $(42 \%)$, pero solo la mitad de las ONL tienen construido un mapa de públicos, lo que en parte explicaría la brecha existente entre la imagen que quieren proyectar las ONL y la imagen percibida por algunos de sus grupos de interés (medios de comunicación, empresas, políticos y sociedad en general).

Asimismo, la investigación de Balas muestra que solo el $35 \%$ de las ONL realiza un plan de comunicación anual o plurianual, lo que indica que existe cierto nivel de planificación enfocado a acciones puntuales y de alto impacto, como campañas de sensibilización social, captación de fondos y de voluntariado, pero carecen de una planificación estratégica de la comunicación a medio plazo. También señala que 
solo el $28 \%$ de las organizaciones encuestadas tienen instrumentos sistemáticos de evaluación de las acciones de comunicación. Finalmente, el estudio de Balas destaca que las acciones de comunicación que más realizan las ONL es publicar contenidos en Internet, seguido por la publicación de folletos y cartelería y la intervención en charlas y conferencias; las ruedas de prensa o entrevistas en medios tienen una frecuencia bastante menor. Los encuestados consideran que las acciones que mejor rentabilizan son las jornadas y seminarios (49\%), la presencia en charlas y conferencias (46\%) y la distribución de folletos y cartelería (35\%).

Otra fuente de interesantes aportaciones es el Anuario del Tercer Sector de Acción Social en España 2012, elaborado por la Fundación Luis Vives sobre una muestra de 716 organizaciones de primer nivel (asociaciones y fundaciones) y de segundo y tercer nivel (federaciones, redes, plataformas y confederaciones). El valor de este informe es que, para algunas variables, ofrece datos desagregados del sector socio-sanitario (146 organizaciones). Este estudio señala que las ONL son conscientes de la necesidad de potenciar su comunicación para proyectar una imagen pública de confianza en el sector y conseguir así una mayor legitimación social pero son pocas las que disponen de los recursos necesarios: buena parte de las ONL disponen de estructuras organizativas con cierto nivel de especialización en administración $(75,3 \%$ ) y gestión de programas y proyectos $(64 \%)$, pero solo el $47,1 \%$ tiene un área de captación de fondos y el $31,4 \%$ de marketing y comunicación externa. La falta de estructuras específicas hace que la presencia pública esté limitada a las organizaciones con más recursos y muchas veces ante situaciones de emergencia o gran impacto mediático.

El informe de la Fundación Luis Vives indica que las ONL otorgan importancia a la comunicación en dos sentidos: en la cooperación entre organizaciones y con instituciones y empresas, y en la realización de campañas de comunicación dirigidas a la captación de fondos y de voluntariado y a dar visibilidad a las acciones que realizan. La tendencia a la interacción entre ONL a través de diversas fórmulas integración (federaciones, plataformas o redes) se traduce, en el caso de las organizaciones socio-sanitarias, en el intercambio de información $(79,6 \%)$, la realización conjunta de campañas $(39,3 \%)$ y el asesoramiento mutuo $(35,8 \%)$.

Asimismo, muestra que el $60,2 \%$ de las ONL afirma realizar habitualmente acciones de comunicación para dar a conocer sus actividades o sensibilizar a la sociedad; ese porcentaje es mayor en las de segundo y tercer nivel $(64,8 \%)$ que en las asociaciones $(61,5 \%)$ y fundaciones $(54,9)$, lo que sin duda habla de posibilidades de construir una estructura de comunicación a través de la agrupación de entidades. En el caso de las ONL del ámbito sociosanitario, los principales objetivos de la comunicación son dar a conocer la entidad $(91,1 \%)$, la sensibilización social $(75,6 \%)$, influir en las administraciones públicas $(39,1 \%)$, recaudar fondos $(38,5 \%)$ y captar socios $(37,9 \%)$.

Este estudio destaca que las ONL combinan la utilización de medios de comunicación convencionales (prensa, radio y televisión), medios sociales y nuevas tecnologías de la comunicación (Internet, webs, mailings, SMS), relaciones públicas (conferencias y charlas) y publicidad. Se aprecia una tendencia a utilizar cada vez más los medios digitales en detrimento de los medios convencionales, aunque estos siguen teniendo un importante papel; en el caso específico de las ONL del ámbito sociosanitario, ya 
son predominantes los medios de comunicación basados en nuevas tecnologías. La gran mayoría de las organizaciones dispone de herramientas de comunicación digital, aunque están más extendidas entre las organizaciones de segundo nivel que entre las asociaciones y fundaciones, con diferencias muy significativas en el uso de medios sociales (Facebook, Twitter y otras redes).

\section{Conclusiones}

El análisis precedente señala la necesidad de desarrollar una línea de investigación que estudie específicamente la comunicación de organizaciones no lucrativas del ámbito de la salud. Como se ha señalado en la introducción de este trabajo, actualmente en España hay activas más de 6.000 ONL del ámbito sociosanitario, pero el conocimiento sobre cómo gestionan su comunicación es todavía escaso por la falta de estudios específicos.

En opinión de este autor, estamos ante un campo de estudio todavía por desarrollar, que debería construirse desde una perspectiva integral que analice en las estrategias, las estructuras, los procesos y las tácticas a través de las cuales esas organizaciones gestionan su comunicación. Para ello, debería incorporar los últimos desarrollos conceptuales en torno a la gestión profesional de la comunicación de las organizaciones, como son las aportaciones de la Nueva Teoría de la Estrategia (Pérez y Massoni, 2008), las relaciones públicas (Grunig y Hunt, 2003; Grunig, 2011; Wilcox, Cameron y Xifra, 2012) y la teoría reputacional (Villafañe, 2004; Villafañe, 2013). Asimismo, estos estudios deberían tomar en cuenta las características particulares y especialización de este tipo de organizaciones.

Estas investigaciones permitirían avanzar en la gestión profesional de la comunicación de las ONL del ámbito de la salud.

\section{Referencias}

Balas, M. (2012). La gestión de la comunicación en el Tercer Sector. Madrid: ESIC Editorial.

Ballvé, J.; Pujol, G.; Romaguera, A.; Bonet, A.; Rafecas, M. y Zarza, E. (2008). Comunicación interna en atención primaria. Atención primaria. Vol. 40, nº 8, pp. 401406.

Beck, C.; Benítez, J.L; Edwards, A.; Olson, A.; Pai, A. y Torres, M. (2004). Enacting "Health Communication": The field of Health Communication as constructed Through Publication in Scholarly Journals. Health Communication. Vol. 16, n. 2, 475-492.

Bustamante, E. (2012). El clima de comunicación, la motivación y la satisfacción laboral en un proceso de atención primaria en Colombia. Revista de Comunicación y Salud. Vol. 3, n 1, pp. 35-49.

Canel, M.J. y Echart, N. (2005). La comunicación de las Fundaciones. En Organización y funcionamiento de las Fundaciones (pp.61-84). Asociación Española de Fundaciones. 
Coe, G. (1998). Comunicación y Promoción de la Salud. Revista Latinoamericana de Comunicación Chasqui. № 63, pp. 26-29.

Costa, C. (2011). La comunicación en el hospital: la gestión de la comunicación en el ámbito sanitario. Sevilla: Comunicación Social ediciones y publicaciones.

Cuesta, U. Menéndez, T. y García, M. L. (2008). Comunicación social y salud: un nuevo planteamiento estratégico. En Cuesta, U. Menéndez, T. y Ugarte, A. (Coords), Comunicación y Salud. Avances en modelos y estrategias de intervención (pp. 19-38). Madrid: Editorial Complutense.

Díaz, H. (2011). La comunicación para la salud desde una perspectiva relacional. En Cuesta, U. Menéndez, T. y Ugarte, A. (Coords), Comunicación y Salud. Nuevos escenarios y tendencias (pp. 33-49). Madrid: Editorial Complutense.

Díaz, H. y Uranga, W (2011). Comunicación para la salud en clave cultural y comunitaria. Revista de Comunicación y Salud. Vol. 1, nº 1. pp. 113-124.

Errasti, F. (2000). Marketing y comunicación en el hospital. En Jiménez, J. (Ed.), Manual de gestión para jefes de servicios clínicos (pp. 85-116). Madrid: Díaz de Santos.

Evans W. (2009). Bibliography. Health Communication. Vol. 24, pp. 186-188.

Freimuth, V.; Massett, H. y Meltzer, W. (2006). ADescriptive Analysis of 10 Years of Research Published in the Journal of Health Communication. Journal of Health Communication. Vol. 11, nº 1, pp. 11-21.

Fundación Luis Vives (2012). Anuario del Tercer Sector de Acción Social en España. Madrid: Fondo Social Europeo.

Grunig, J. y Hunt, T. (2003). Dirección de relaciones públicas. Edición adaptada al castellano por Jordi Xifra. Barcelona: Ediciones Gestión 2000.

Grunig, J (2011). Characteristics of Excellent Communication. En Gillis, T (Ed.), The IABC Handbook of Organizational Communication. San Francisco: IABC.

Herranz de la Casa, J.M. (2007). La gestión de la comunicación como elemento generador de transparencia en las organizaciones no lucrativas. CIRIEC-España, Revista de Economía Pública, Social y Cooperativa. Nº 57 , pp. 5-31.

Hill, S. (2009). Directions in health communication. Bulletin of World Health Organization. No 87, pp. 648. Ginebra, WHO.

Instituto Nacional del Cáncer de los Estados Unidos (2004). Making health communication programs work. Disponible en http://www.cancer.gov/pinkbook. Recuperado el $10 \mathrm{de}$ febrero de 2009.

Kim, J.; Park, S.; Yoo, S. y Shen, H. (2010) Mapping Health Communication Scholarship: 
Breadth, Depth, and Agenda of Published Research in Health Communication. Health Communication. Vol. 25, $\mathrm{n}^{\circ}$ 6-7, pp. 487-503.

Kreps, G. y Maibach, E. (2008). Transdisciplinary Science: The Nexus Between Communication and Public Health. Journal of Communication. Vol. 58, pp. 732-748.

Lalonde, M (1974). A new perspective on the Health of Canadians. Ottawa: Ministery of Health Care and Welfare of Canada. Disponible en http://www.fundadeps.org/Observatorio. asp?codrecurso=132. Recuperado el 15 de junio de 2013.

March, J.C.; Prieto, M. y Gutiérrez, P. (2000). Profesionales y gestores ante la comunicación interna en el Programa de Salud Materno infantil de Andalucía. Atención Primaria. Vol. 25, $n^{\circ} 1$, pp 16-21.

March, J. C.; Prieto, M.A.; Pérez, O.; Minué, S. y Danet, A. (2011). La comunicación interna en centros de Atención Primaria en España. Revista de Comunicación y Salud. Vol. 1, $\mathrm{n}^{\circ}$ 1, pp. 18-30.

Medina Aguerrebere, P. y González Pacanowsky, A. (2011). Comunicación de la salud: una aproximación multidisciplinar. Madrid: Fragua.

Medina Aguerrebere, P. (20121). El valor estratégico de la comunicación interna hospitalaria. Revista de Comunicación y Salud. Vol. 2, nº 1, pp. 19-28.

Medina Aguerrebere, P. $\left(2012^{2}\right)$. La responsabilidad social corporativa en hospitales: un nuevo desafío para la comunicación institucional. Revista Española de Comunicación en Salud. Vol. 3, n 1, pp. 77-87.

Muñoz-Cruzado y Barba, M. (2010). La comunicación en la salud. Revista Española de Comunicación en Salud. Vol. 1, número 2: pp 66-67.

Ministerio del Interior del Gobierno de España (2012). Anuario Estadístico 2011 del Ministerio del Interior. Disponible en http://www.interior.gob.es/file/58/58114/58114.pdf. Recuperado el 10 de junio de 2013.

Missoni, E. (2012). Rol y contribución de las organizaciones no gubernamentales y de la sociedad civil en la promoción de la salud global. En Juvinyà, D y Arroyo, H (Eds), La Promoción de la Salud 25 años después. Publicaciones de la Cátedra de Promoción de la Salud de la Universidad de Girona. Girona. Documenta Universitaria.

Nazione, S.; Pace, K.; Russell, J. y Silk, K. (2013). A 10-Year Content Analysis of Original Research Articles in Health Communication and Journal of Health Communication (20002009). Journal of Health Communication. Vol. 18, pp. 223-240.

Obregón, R. (2010). Un panorama de la investigación, teoría y práctica de la comunicación en salud. Revista Folios. N 23, pp. 13-29.

Organización Mundial de la Salud (1986). Carta de Ottawa para la Promoción de la Salud. Ginebra, OMS. Disponible en http://www1.paho.org/spanish/hpp/ottawachartersp.pdf. Recuperado el 20 de mayo de 2013. 
Organización Mundial de la Salud (2001). Alianzas estratégicas. El papel de la sociedad civil en el ámbito de la salud. Documento de trabajo, 1, 2001, CSI/2001/DP1. Ginebra: OMS. Disponible en http://www.who.int/civilsociety/documents/en/AlianzasEstrategicas2001DP1-sp.pdf. Recuperado el 20 de mayo de 2013.

Organización Mundial de la Salud OMS (2006). Constitución de la OMS. Documentos básicos. Disponible en http://www.who.int/governance/eb/who_constitution_sp.pdf. Recuperado el 20 de mayo de 2013.

Organización Panamericana de la Salud (2001). Manual de comunicación social para programas de promoción de la salud de los adolescentes. Washington, Fundación W.K. Kellogs.

Pérez, R.A. y Massoni, S. (2008). Hacia una teoría general de la estrategia. Barcelona: Ariel.

Petracci, Mónica y Waisbord, Silvio (2011) (Coords.). Comunicación y salud en la Argentina. Buenos Aires, La Crujía.

Salamón, L. y otros (2001). La sociedad civil global. Las dimensiones del sector no lucrativo. Bilbao. Fundación BBVA.

Saló, N. y Clèries, X. (2007). La auditoría de comunicación. Una herramienta para las organizaciones sanitarias. Revista de Administración Sanitaria. Vol. 5, n¹, pp. 123-135.

Terrón, J.L. (2010). Algunas reflexiones sobre comunicación y salud en España tras cinco años del Observatorio de Comunicación y Salud. Revista Española de Comunicación en Salud. Vol. 1, n² 2, pp. 77-97.

Thompson, T.L. (2006). Seventy five Issues if Health Communication: An Analysis of Emerging Themes. Health Communication. Vol. XX, nº 2, pp. 117-123.

Ugarte, A. (2008). El binomio comunicación y salud: relaciones e investigación. En Cuesta, U. Menéndez, T. y Ugarte, A. (Coords), Comunicación y Salud. Avances en modelos y estrategias de intervención (pp. 39-47). Madrid: Editorial Complutense.

Vernis, A.; Iglesias, M.; Sanz, B.; Solernou, M.; Urgell, J. y Vidal, P. (1998). La Gestión de las Organizaciones No Lucrativas. Bilbao: Deusto.

Villafañe, J. (2003). La buena reputación. Claves del valor intangible de las empresas. Madrid: Pirámide.

Villafañe, J. (2013) La buena empresa. Propuesta para una teoría de la reputación corporativa. Madrid: Pearson Educación.

Wilcox, D. Cameron, G. y Xifra, J. (2012). Relaciones públicas. Estrategias y tácticas. Madrid: Pearson Educación. 